\title{
Tumor Therapy with Amanita phalloides (Death Cap): Stabilization of B-Cell Chronic Lymphatic Leukemia
}

\author{
Isolde Riede, PhD
}

\begin{abstract}
Background: Molecular events that cause tumor formation upregulate a number of HOX genes, called switch genes, coding for RNA polymerase II transcription factors. Thus, in tumor cells, RNA polymerase II is more active than in other somatic cells. Amanita phalloides contains amanitin, inhibiting RNA polymerase II. Partial inhibition with amanitin influences tumor cell—but not normal cell—activity.

Objectives: To widen the treatment spectrum, homeopathic dilutions of Amanita phalloides, containing amanitin, were given to a patient with leukemia. Monitoring the leukemic cell count, different doses of amanitin were given.

Results: The former duplication time of leukemic cells was 21 months. Within a period of 21 months, the cell count is stabilized to around $10^{5} / \mu \mathrm{L}$. No leukemia-associated symptoms, liver damage, or continuous erythrocyte deprivation occur.

Conclusions: This new principle of tumor therapy shows high potential to provide a gentle medical treatment.
\end{abstract}

\section{Introduction}

$\mathbf{I}_{\mathrm{s}}^{\mathrm{N}}$ N A GENETIC ATTEMPT to clarify pathways and find central genes with tumor-forming fly Drosophila melanogaster, four classes of genes involved in tumor formation have been distinguished.

Proliferative genes break the cell cycle control by allowing replication immediately after mitosis. They influence the somatic pairing of chromosomes. ${ }^{1}$ One single proliferative mutation is sufficient for tumor formation. These mutations lead to intrinsic chemotherapy resistance, and to replication partially with the repair system. ${ }^{2}$ Mutations in oncogenes and tumor-suppressor genes are secondary events and add by destabilization of the differentiation pattern of the cells. Neither oncogene nor tumor-suppressor gene mutations alone or in combinations lead to tumor formation. ${ }^{3}$

The variation of oncogene, tumor-suppressor gene, and proliferative gene mutations in tumor formation is complex. By crossing D. melanogaster, all genes involved were scanned for interaction. A target for tumor therapy should interact with a number of tumor-forming gene combinations (i.e., should reveal lethality over tumor-forming gene combinations and survival over wild type).

In search for ubiquitous events-the central possible targets for therapeutic intervention-switch genes were identified: They interact with tumor-forming mutations in trans. A mutation within a switch gene is lethal over several on- cogene, tumor suppressor gene or proliferative gene mutations, or combinations thereof. Switch genes are usually not mutant in tumors. ${ }^{4}$ Figure 1 summarizes the pathway of genes involved in tumor formation. All switch genes belong to the class of $H O X$ genes, and interfere with a large number of other genes during their action in development. Switch genes also change chromatin structure, and inhibit somatic pairing of chromosomes. Functionally, switch genes all code for RNA polymerase II (RNAP) transcription factors (Fig. 2).

Some HOX genes are overexpressed in human tumor cells. ${ }^{5,6}$ Due to this overexpression, the event that leads to tumor cell growth should lead to high activity of RNAP. In somatic cells of adults, RNAP is expected to be less active. Partial inhibition of this enzyme consequently causes inhibition of tumor cell activity, without severe effects on somatic cells. The drug in the extract of Amanita phalloides, amanitin, blocks RNAP in all cells. Inhibition of about $50 \%$ of this molecule has no effect on normal body cells. ${ }^{7}$

Within a lifespan, several tumor cells arise in the body. Not every mutation leading to a tumor cell develops into a massive tumor. Immune cells usually recognize and lyse tumor cells. Only if the tumor cells grow faster that the immune system can digest them will a cancer disease be established. An expedient tumor therapy is based upon two premises. First, the number of tumor cells should be reduced, without affecting somatic cells, especially immune cells. Second, the immune systems require strengthening. 
FIG. 1. Genetic pathway in tumor formation. Switch genes (bolt) are the central interaction point of all genes that contribute to tumor formation. This pathway is the summary of Drosophila genetics, analyzing the events of a brain tumor formation. Interacting genes show mutations, that are lethal in trans.

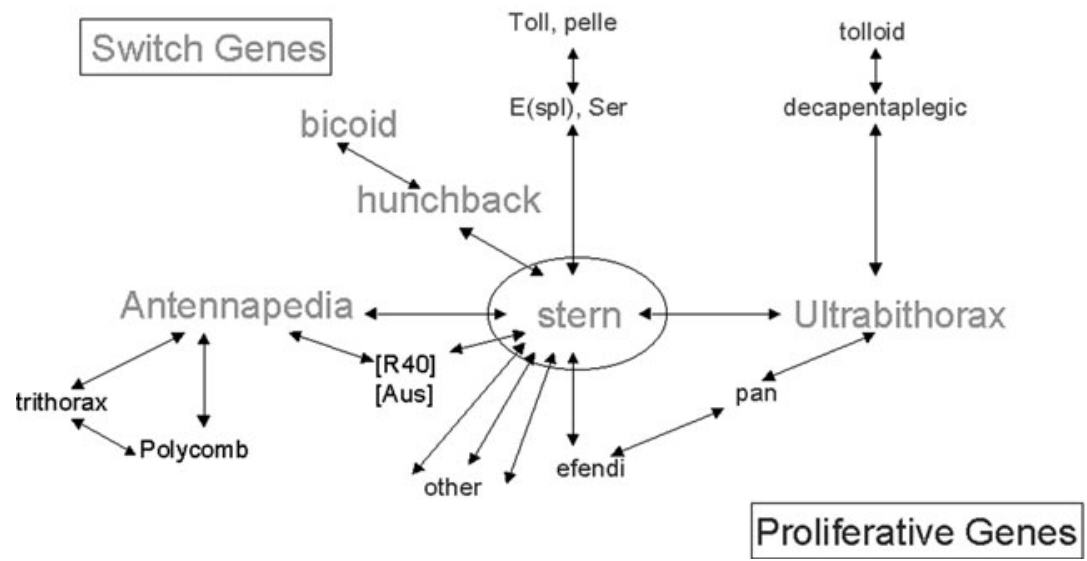

In the classical sense, homeopathic dilutions of $A$. phalloides are used as a remedy against fear of death. In the molecular sense, this remedy has been newly discovered to have activity as an inhibitor specific for tumor cells. The immune system might recognize and lyse tumor cells, and partial inhibition of tumor cell activity raises the possibility for stabilization of the disease. Amanita treatment already showed good results in prophylaxis or cure of a number of tumor cases. To expand the possibilities of experimental medicine, a pilot study of treatment of B-cell chronic lymphatic leukemia (B-CLL) is described here. As outlined previously, ${ }^{8,9}$ the therapy was administered with $A$. phalloides (zert. Riede) homeopathic dilutions.

\section{Patient and Methods}

The patient was born in 1957 and works in the field service. B-CLL was first diagnosed in 2005, appearing to be slowly progressive. Amanita therapy started at the end of 2007 at a cell count of $1.26 \times 10^{5}$ leukocytes per $\mu \mathrm{L}$ blood. This

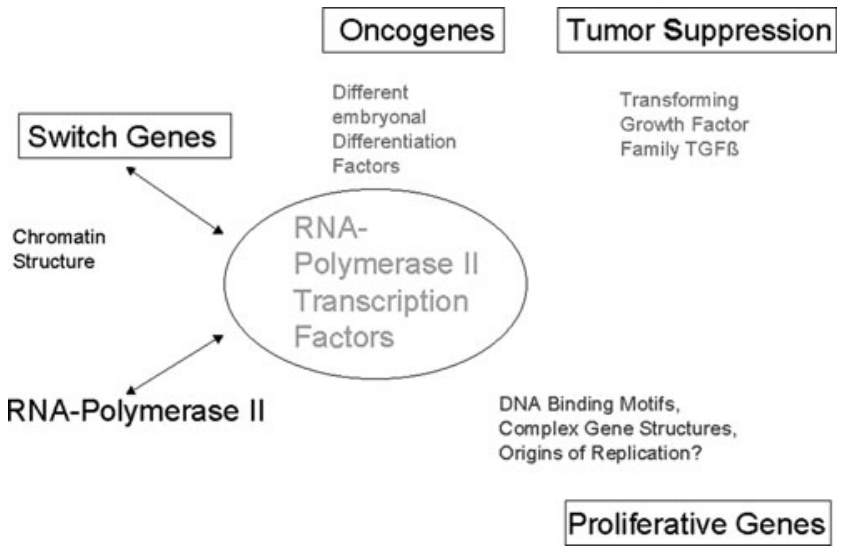

FIG. 2. Biochemistry of tumor formation. All switch genes are transcription factors for RNA polymerase II (RNAP). Overexpression of switch genes lead to $100 \%$ activity of RNAP in tumor cells. In normal cells, the enzyme activity is lower. Partial inhibition of activity leads to tumor cell activity reduction without affecting normal cells. leukocyte level is too low for conventional treatment. The patient decided to undertake a therapy attempt with Amanita, until a level of $2 \times 10^{5}$ leukocytes per $\mu \mathrm{L}$ blood was achieved or until liver cell damage appeared. In parallel, all prearrangements for conventional therapy occurred. The patient's history did not indicate any specific risk for tumor formation. His body weight was $77 \mathrm{~kg}$ and remained constant throughout the therapy. No additional disease was diagnosed. He subsists on vegetarian food and often suffers from low iron levels. He shows no symptoms of leukemia, no lymph node swelling, and does not have frequent infections. The erythrocyte cell count is $4.5 \times 10^{6} / \mu \mathrm{L}$ blood, which is at a low level within the normal range.

No other tumor-specific therapy was applied. Adjuvant uptake of additional essential fatty acids (to enhance fluidity of cell membranes and to provide raw material for prostaglandins, i.e., immune cell hormones), iron (orally), and zinc (cutaneous) was given. Noxes (i.e., long airplane flights or radiation) are avoided as far as possible.

A minimum dose of 5 drops of $A$. phalloides (zert. Riede) D4 (herbamed AG) per day is usually applied to prevent growth of residual tumor cells. A maximum dose of $4 \times 10$ drops D2 per day is applied for fast-growing and/or large tumor masses, usually without side-effects. The intake per linguale ensures an easy passage of the drug to the most metabolically active cells through the oral mucosa.

For monitoring of the therapy, the following parameters were regularly measured:

1. Lactate dehydrogenase (LDH) in serum. This enzyme is present in all cells, and its occurrence in serum shows lysis of cells. LDH levels increase in all events, resulting in cell degradation (i.e., myocardial or kidney infarction, embolism, autoimmunity, anemia, infectious diseases, or lysis of tumor cells).

2. Cell count of leukocytes in blood for monitoring of tumor cell growth or cellular lysis or migration activity.

3. Differential blood count for monitoring occurrence of anemia.

4. Liver enzymes $\gamma$-glutamyltransferase (GGT), glutamicoxaloacetic transaminase (GOT), and glutamic-pyruvic transaminase (GPT), which occur in serum upon lysis or 
damage of liver cells, for monitoring possible intoxication of the liver with amanitin.

\section{Results}

The initial exponential growth of leukocytes reveals a period of 21 months for duplication. The progress of the amanita therapy is outlined over one duplication time.

\section{1-6 months of therapy}

The initial amanitin dose at a cell count of $1.26 \times 10^{5} / \mu \mathrm{L}$ is low, $3 \times 15$ drops of $A$. phalloides D4 per day. This leads to reduction of the growth rate of the cells (Fig. 3). To stabilize and reduce the number of leukocytes, $1 \mathrm{~mL}$ D2 (i.e., 10 drops per day) is applied. This leads to a reduction of leukocytes.

\section{5 months of therapy}

At a cell count of $0.78 \times 10^{5} / \mu \mathrm{L}, \mathrm{LDH}$ increases, revealing cellular lysis. Cervical lymph nodes swell, fever and night sweats occur, hands and fingers are edemic, and the patient is thirsty. The symptoms reveal lymphatic inflammation.

\section{8 months of therapy}

At a leukocyte cell count of $0.45 \times 10^{5} / \mu \mathrm{L}, \mathrm{LDH}$ is still high, revealing lysis of cells. Fever and night sweat continue, and lymph nodes are shrunken. The GPT value is higher, but still within the average range. GGT and GOT remain constant. The erythrocyte count is at $4.1 \times 10^{6} / \mu \mathrm{L}$ blood. Amanita uptake was interrupted.

\section{A week later}

The leukocyte count is back to $1.08 \times 10^{5} / \mu \mathrm{L}$, and the erythrocyte count is low at $3.4 \times 10^{6} / \mu \mathrm{L}$ blood.

\section{9 months of therapy}

At this stage, the leukocyte level remains constant, LDH decreases, and cell lysis ceases. The patient no longer shows symptoms. The erythrocyte count is back to normal range at $4.5 \times 10^{6} / \mu$ L blood.

\section{9-19 months of therapy}

Amanita uptake interrupted by breaks lead to a zigzag increase of leukocytes followed by a decrease coupled with the increase of LDH (i.e., lysis of cells). The erythrocyte count remains around $4.5 \times 10^{6} / \mu \mathrm{L}$ blood. Reductions of leukocytes are now maintained, without inflammation symptoms.

\section{0 months of therapy}

At a level of $2 \times 10^{5} / \mu \mathrm{L}$ leukocytes, the erythrocyte count drops to $3.7 \times 10^{6} / \mu \mathrm{L}$ blood. A high dose of amanita is applied. The leukocyte cell count drops within 2 weeks to $1.4 \times 10^{5} / \mu \mathrm{L}$ blood, followed by an increase of LDH and a recovery of the erythrocyte level to $4.5 \times 10^{6} / \mu \mathrm{L}$ blood.

Liver enzymes remain in the average range all the time. The patient is tired and exhausted.

At the beginning of therapy, $1.26 \times 10^{5}$ leukocytes $/ \mu \mathrm{L}$ blood occurred, after a duplication time of 21 months $2.52 \times 10^{5}$ leukocytes/ $\mu \mathrm{L}$ blood would have been expected. With the amanita therapy, a value of $1.43 \times 10^{5} / \mu \mathrm{L}$ is achieved, indicating that over a duplication time the cell count could be kept relatively constant, presumably elongating the lifespan of the patient without severe side effects.

\section{Discussion}

Leukemia appears as disease that is often diagnosed as anemia: lack of erythrocytes. The tumor growth of leukocytes replaces over time the erythropoietic stem cells in the bone marrow system and leads to erythrocyte deprivation. Here a study is presented applying a new principle for tumor therapy: Amanitin treatment. With amanitin, the activity of tumor cells is retarded.

Monitoring leukocyte appearance in the blood leads to dose administration. Whenever the cell count increases, and the general condition of the patient is best, a higher dose is applied and vice versa. Every cycle of a high dose (i.e., $80 \mathrm{~mL}$ of D2 in a few months) started at a low erythrocyte level.

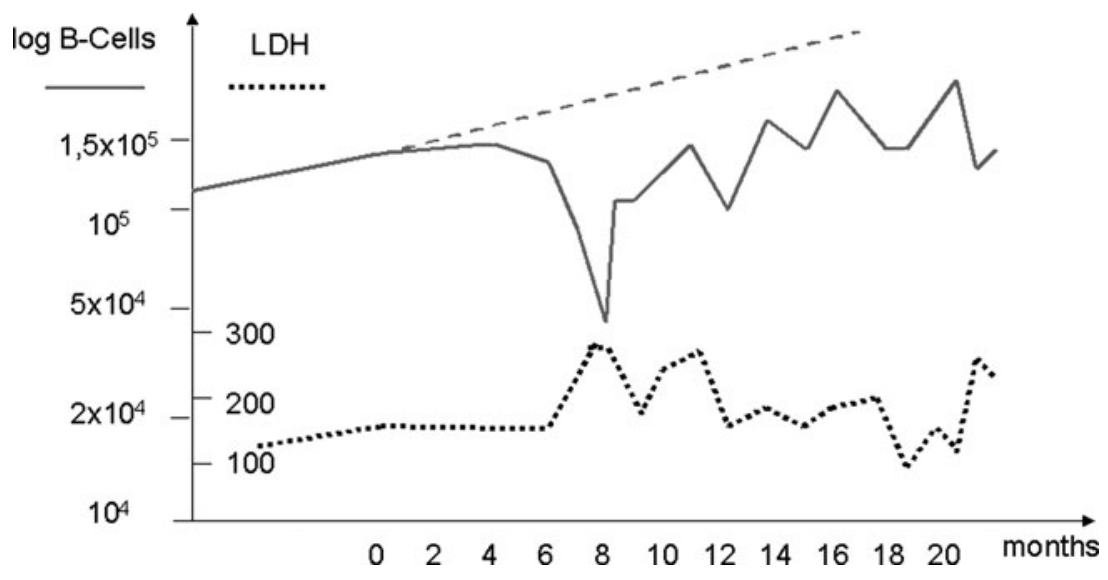

days, followed by $5 \times 15$ drops $14 / \mathrm{d}$ in total $95 \mathrm{~mL} D 2$ incorporated; $11-12$ mont D2 incorporated; $8-10$ months break of 5
ths $4 \times 10$ drops/day, in total $130 \mathrm{~mL} \mathrm{D} 2$ incorporated; 13-15 months break; 16-17 months $4 \times 10$ drops D2/d, in total $155 \mathrm{~mL}$ D2 incorporated; 18 months break of 2 weeks, followed by $3 \times 5$ drops/d; 19 months break; 20-21 months $6 \times 6$ drops D2/d, in total 180 mL D2 incorporated. Thrombocytes remain constant in the average range all the time.

FIG. 3. Treatment of B-cell chronic lymphatic leukemia (B-CLL). Outlined are the values of the leukocyte cell count in blood (Log B-cells [/ $\mu \mathrm{L}$ blood]) over time (solid line). Before amanita therapy, the cells show exponential growth with a duplication time of 21 months (elongated as broken line). At time zero, amanita therapy started. Lactate dehydrogenase $(\mathrm{LDH})$ values (dotted line) alternate with leukocyte count. Whenever a high dose is applied, leukocyte values drop and LDH values rise, revealing lysis of tumor cells. Dosage: $0-3$ months $3 \times 15$ drops D4/d, equivalent to $7 \mathrm{~mL}$ D2 incorporated; $4-7$ months $4 \times 10$ drops $\mathrm{D} 2 / \mathrm{d}$, in total $82 \mathrm{~mL}$ D2 incorporated; 8-10 months break of 5 
Due to amanitin uptake, the leukocyte level in the blood decreases. Some cells lyse, appearing with LDH increase. This leads repeatedly to recovery of the erythrocyte level in the blood within a short time.

Without amanitin, the LDH levels remain within the normal range, indicating that the immune system does not efficiently lyse tumor cells. Upon amanitin uptake, the leukocyte cell count ceases and lysis of cells occur, indicating that presumably tumor leukocytes lyse. This lysis shows that amanitin might not only interrupt the activity of tumor growth, but could alter antigen expression, and thus the immune system might attack the tumor cells more efficiently.

Eight (8) months after the beginning of therapy, a decrease of the leukocyte count to $0.45 \times 10^{5} / \mu \mathrm{L}$ is achieved, coinciding with symptoms of lymphatic inflammation. Within a week, the leukocyte level increases to $1.08 \times 10^{5} / \mu \mathrm{L}$. This duplication is not expected to result from cell growth; the duplication time is 21 months. Alternately, migration of leukocytes is discussed. Coincidentally, the decrease of leukocyte count in blood might occur not only from lysis of cells, but also from migration to the periphery. Thus, amanitin might influence the migration of leukocytes.

Later applications of high doses do not lead to symptoms of lymphatic inflammation. It can be expected that at the beginning, the cells are synchronized concerning their uptake of amanitin, all migrating or lysing at once, causing symptoms of inflammation. After a while, cells could be in different stages concerning their amanitin content, smoothing the clinical symptoms of migration and lysis, and resulting in symptoms of general exhaustion.

With approximately $1-200 \mathrm{~mL}$ of $A$. phalloides D2 per year, the tumor growth of B-CLL can be arrested. Amanita therapy also shows good results in therapy or prophylaxis in a variety of other tumors such as colon carcinoma, mammary carcinoma, or tongue root tumor. 7,9

This therapy does not affect the activity of somatic cells, especially the immune cells. Amanitin stops the activity of the tumor cells, and then they lyse and migrate. Thus, ho- meopathic dilutions from $A$. phalloides offer a strong tool for the therapy of cancer.

\section{Disclosure Statement}

No competing financial interests exist.

\section{References}

1. Riede I. Proliferative genes induce somatic pairing defects in Drosophila melanogaster and allow replication. Canc Genet Cytogenet 1997;97:143-154.

2. Riede I. Tumor inducing Drosophila: Resistance to hydroxyurea and methane sulfonic acid methyl ester. Drosophila Information Service 2000;83:140-144.

3. Riede I. Gene combinations inducing neoplasms in Drosophila. Drosophila Information Service 2002;84:103-114.

4. Riede I. Switch Genes in Tumor Formation. Drosophila Information Service 2004;87:54-59.

5. Rozovskaia T, Feinstein E, Mor O, et al. Upregulation of Meis1 and HoxA9 in acute lymphocytic leukemias with the $\mathrm{t}(4: 11)$ abnormality. Oncogene 2001;20:874-878.

6. Hudlebush HR, Lodahl M, Johnsen HE, Rasmussen T. Expression of HOXA genes in patients with multiple myeloma. Leuk Lymphoma 2004;45:1215-1217.

7. Riede I. The biochemistry of the tumor cell [in German] Naturheilpraxis 2007;12:1733-1743.

8. Riede I. Novel tumor therapy [in German]. Naturheilpraxis 2005;3:342-343.

9. Riede I. The management of the tumor disease [in German]. Naturheilpraxis 2008;9:12-19.

Address correspondence to: Isolde Riede, $\mathrm{PhD}$ Im Amann 7 Ueberlingen D-88662 Germany

E-mail: riede@tumor-therapie.info 